\title{
Modularity of a Cambrian ptychoparioid trilobite cranidium
}

\author{
Mark Webstera,* and Miriam L. Zelditch \\ ${ }^{a}$ Department of the Geophysical Sciences, University of Chicago, 5734 South Ellis Avenue, Chicago, IL 60637, USA \\ ${ }^{\mathrm{b}}$ Museum of Paleontology, University of Michigan, Ann Arbor, MI 48109-1079, USA \\ *Author for correspondence (email: mwebster@geosci.uchicago.edu)
}

\begin{abstract}
SUMMARY Modularity of the cranidium of Crassifimbra? metalaspis, a Cambrian ptychoparioid trilobite, is investigated using landmark-based geometric morphometric methods to gain insight into the integration among morphogenetic processes responsible for shaping the head of an ancient arthropod. Of particular interest is the extent to which the structure of phenotypic integration was governed by direct interactions among developmental pathways, because these interactions may generate long-term constraints on evolutionary innovation. A modified two-way ANOVA decomposes cranidial shape variation into components representing symmetric variation among individuals and fluctuating asymmetry (FA). The structure of integration of each of these components is inferred from correlated deviations in shape among nine partitions of the cranidium. Significant correlation
\end{abstract}

among partitions in FA indicates direct interactions among their respective developmental pathways. An a priori hypothesis that modularity was determined by functional association among partitions is not well supported by the among-partition correlation structure for either component of variation. Instead, exploratory analyses reveal that phenotypic integration was strongly influenced by spatially localized morphogenetic controls. Comparison of the structures of the Individuals and FA components of variation reveals that the two share relatively few commonalities: the structure of phenotypic integration was only weakly influenced by direct interactions. The large contribution of parallel variation to phenotypic integration suggests that modularity was unlikely to have imposed a long-term constraint on evolutionary innovation in these early trilobites.

\section{INTRODUCTION}

Phenotypic integration is expected to arise when traits are functionally or developmentally coupled within individuals; according to long-standing theory, integration at the individual level is expected to lead to phenotypic integration at population level, that is manifest by statistical correlations between traits (Olson and Miller 1958; Cheverud 1982, 1984; Schlosser and Wagner 2004). Such integration channels variation into a few dimensions, and leads to the recognition of variational modules (i.e., suites of traits that covary more highly with each other than with traits belonging to other modules). Depending on its congruence with selective pressure, the structure of integration (and thus variational modularity) can constrain the direction and either impede or enhance the rate of evolution (Simpson 1944; Burger 1986; Wagner 1988; Schluter 1996; Wagner and Altenberg 1996; Marroig and Cheverud 2005; Renaud et al. 2006; Sniegowski and Murphy 2006; Hunt 2007).

The lability of phenotypic integration determines the extent to which it may affect macroevolutionary diversification. If integration itself rapidly evolves, then directions of "least resistance" can also change rapidly, making integration only a short-term constraint on innovation. An important and novel hypothesis proposes that the developmental cause of integra- tion may determine how rapidly integration itself evolves. Two kinds of developmental causes of correlations have been distinguished: those caused by (1) parallel variation, whereby the same source of variation acts independently in two or more developmental modules; and (2) direct interactions, whereby variation is transmitted within and between developmental pathways that directly connect to each other such as by partitioning of material or signaling (e.g., Klingenberg and Zaklan 2000; Klingenberg et al. 2001, 2003; Klingenberg 2005). It is hypothesized that correlations can be restructured more easily if they arise from parallel variation than from direct interactions because the former can occur by selection for favorable patterns of pleiotropy, whereas the latter requires restructuring of developmental pathways (Klingenberg 2005). Thus, to the extent that integration is due to direct interactions, it is likely to be temporally conservative except for rare, rapid changes in structure. Lability of phenotypic integration is almost invariably inferred by comparison of the structure of integration among extant organisms. Variational modularity of extinct species has rarely been studied, although paleontological examples were a key component of the pioneering work by Olson and Miller (1958). The contribution of direct interactions to phenotypic integration is known for only a few taxa, all extant (e.g., Klingenberg and Zaklan 2000; Klingenberg et al. 2001, 2002, 2003, 2004; Klingenberg 2004, 
2005; Zelditch et al. 2008, 2009; Drake and Klingenberg 2010). As a result, it is difficult to say whether temporal trends in rates and directions of diversification, as revealed by the fossil record, can be ascribed to direct interactions.

Here we investigate the structure of integration of a Cambrian trilobite, with the specific aims of (1) gaining insight into the developmental underpinning of shape variation in an ancient arthropod, and (2) determining the extent to which the structure of phenotypic integration was governed by direct interactions and was thus likely to have served as a long-term constraint on innovation. We use as a case study Crassifimbra? metalaspis (Sundberg and McCollum 2000), an early ptychoparioid trilobite from the uppermost Dyeran (traditional Laurentian "Lower Cambrian") Pioche Formation of Nevada (Webster in press). Early ptychoparioid trilobites are thought to be the plesiomorphic ancestral stock from which more derived trilobite clades arose (Fortey in Whittington et al. 1997, p. 296; Fortey 2001, p. 1148) and thus occupy a pivotal place in trilobite evolution. Our study focuses on the cranidium (Figs. 1 and 2), a morphologically complex cephalic sclerite well suited for analysis of shape using landmarks and semilandmarks. A variety of structures are incorporated into the cranidium, associated with different functions (Whittington et al. 1997; below). A modular arrangement of parts within such a multifunction sclerite might be expected. An understanding of cranidial modularity is important because modification of cranidial shape was a major component of trilobite evolution (Foote 1989, 1990, 1991, 1993a, b; Fortey and Owens in Whittington et al. 1997).

Our article is structured as follows: we first present an a priori model for the functional integration of the cranidium; unlike the developmental and genetic regulators of form, functional attributes of cranidial regions are reasonably wellknown. Because we have only one a priori hypothesis and that might not be the optimal model for cranidial integration, we follow the test of the functional model by exploratory analyses, using these to derive an a posteriori model of cranidial integration. We then assess the contribution that direct interactions made to phenotypic integration.

\section{Functional integration of the cranidium}

The function of the various structures incorporated into the cranidium is reasonably well established (Whittington et al. 1997): the glabella covered the stomach and anterior portion of the digestive tract, and was also the site of attachment for cephalic appendages; the palpebral lobes were associated with the eye; the facial sutures were associated with ecdysis; and the posterior cranidial margin was associated with articulation between the cranidium and anterior most thoracic segment. This permits development of a simple model for the predicted functional integration of the cranidium. The glabella, the palpebral lobes, the facial sutures, and the posterior cranidial margin are expected to represent distinct modules, based on their differing functions (associated with digestion/appendage attachment, the visual field, ecdysial mechanics, and cephalontrunk articulation mechanics, respectively). The anterior cranidial margin is assigned to the ecdysial functional module given its close association with the rostral suture, which lies subparallel and immediately interior to the anterior margin on the ventral surface (Fig. 1, B, G, and K). This simple functional model predicts that the module relating to the visual field is decoupled from that relating to ecdysial mechanics. However, the distal margin of the palpebral lobes is also defined by an ecdysial suture. This article does not develop more complex functional models that involve integration between these hypothesized modules (but see exploratory analyses, below).

To assess the fit of the functional model to the data, we divide the cranidium into nine partitions, and represent the expected correlations among those partitions (Fig. 2C) by edges between nodes of a graph. In specifying the model we distinguish between edges that are left free to be estimated by maximum-likelihood and those that are fixed by the hypothesis; the ones that we fix are between hypothesized modules - these are expected to be conditionally independent. The fit of the model to the data is then assessed by a $\chi^{2}$-value, which is a function of deviation between the model and data and the sample size. We also use the Akaike Information Criterion (AIC) to determine whether the hypothesis of functional integration improves upon the null model of complete integration (i.e., the saturated model). The AIC is $2 k-2 \ln (L)$, where $k$ is the number of parameters and $L$ is the maximized value of the likelihood function (Akaike 1974); the model with the lower AIC is therefore preferred. We also evaluate fit of the functional model by constructing a target matrix in which partitions hypothesized to belong to the same module are assigned a correlation of 1.0 and partitions hypothesized to belong to different modules are assigned a correlation of zero; the matrix correlation between this target matrix and the observed among-partition correlation matrix is then tested for its statistical significance using the permutation-based Mantel test (Mantel 1967; Dietz 1983). This technique is regularly used to compare correlation matrices (e.g., Kohn and Atchley 1988; Cheverud et al. 1989, 1991; Cheverud 1995; Ackermann and Cheverud 2000; Hallgrímsson et al. 2004; Young 2004; Monteiro et al. 2005; Zelditch et al. 2008, 2009).

\section{MATERIAL AND METHODS}

\section{Material}

Silicified sclerites of Crassifimbra? metalaspis (Fig. 1) were recovered from a thin carbonate bed approximately $10.75 \mathrm{~m}$ above the base of the Combined Metals Member (Pioche Formation) at the Log Cabin Mine section, Highland Range, east-central Nevada (sample ICS-10124; Webster in press). Taphonomic and stratigraphic data 


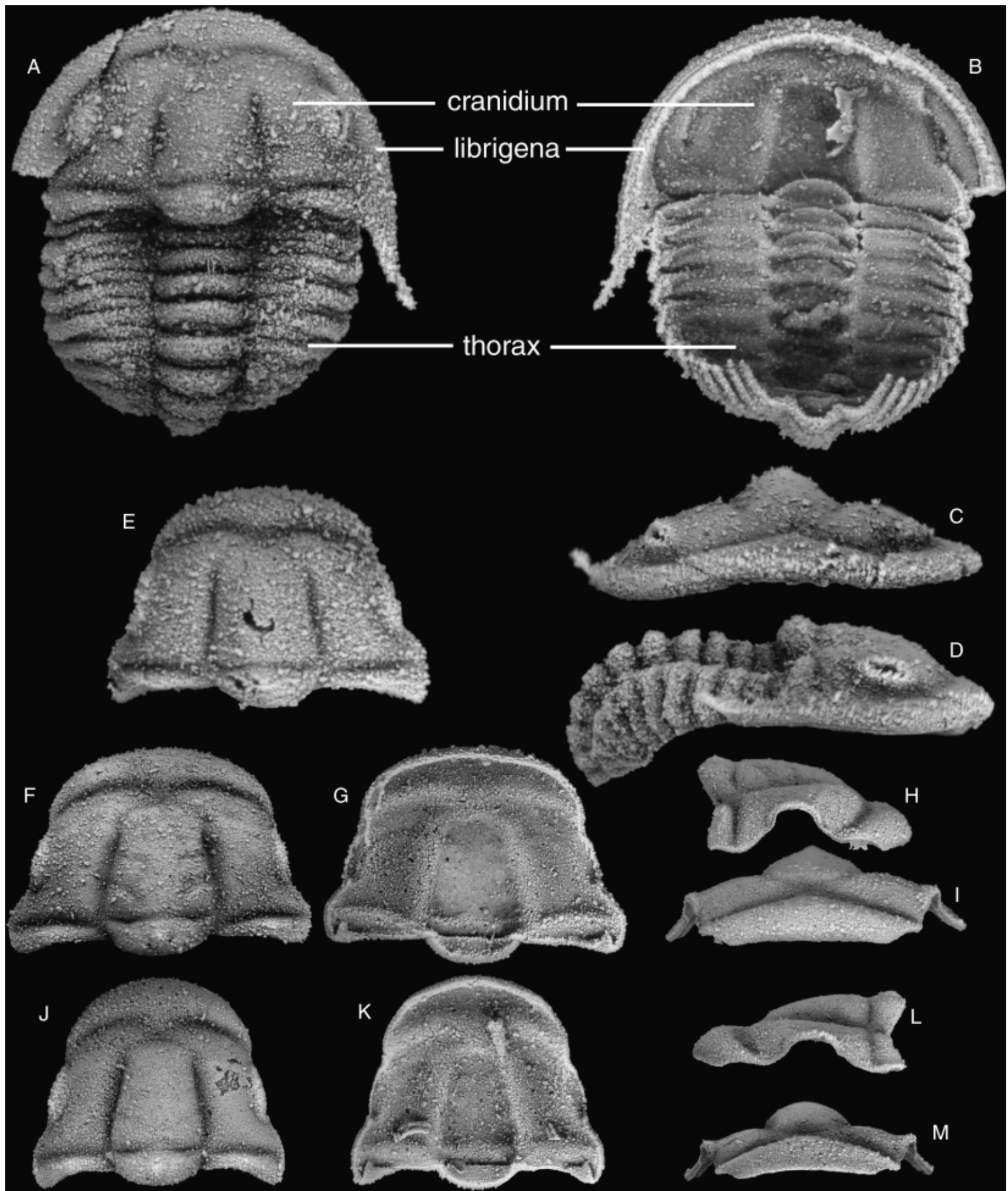

Fig. 1. Representative silicified specimens of Crassifimbra? metalaspis. (A-D) Articulated dorsal exoskeleton with 14 thoracic segments (pygidium missing) in dorsal, ventral, anterior, and right lateral views, FMNH PE58337, $18 \times$. The cranidium and librigena, separated by the facial suture, together comprise the cephalon (head shield). (E) Small cranidium in dorsal view, FMNH PE58278, $22 \times$. (F-I) Large cranidium in dorsal, ventral, right lateral, and anterior views, FMNH PE58271, $9 \times$. (J-M) Large cranidium in dorsal, ventral, right lateral, and anterior views, FMNH PE58273, $9 \times$. All from ICS-10124, Combined Metals Member, Pioche Formation, Log Cabin Mine section, Highland Range, Nevada. 
indicate that this bed accumulated over just decades to hundreds of years and therefore contains a minimally time-averaged fossil assemblage (Webster in press). The sclerites are typically in excellent condition, suggesting minimal transportation and reworking. This bed is of latest Dyeran age, approximately 510 million years old
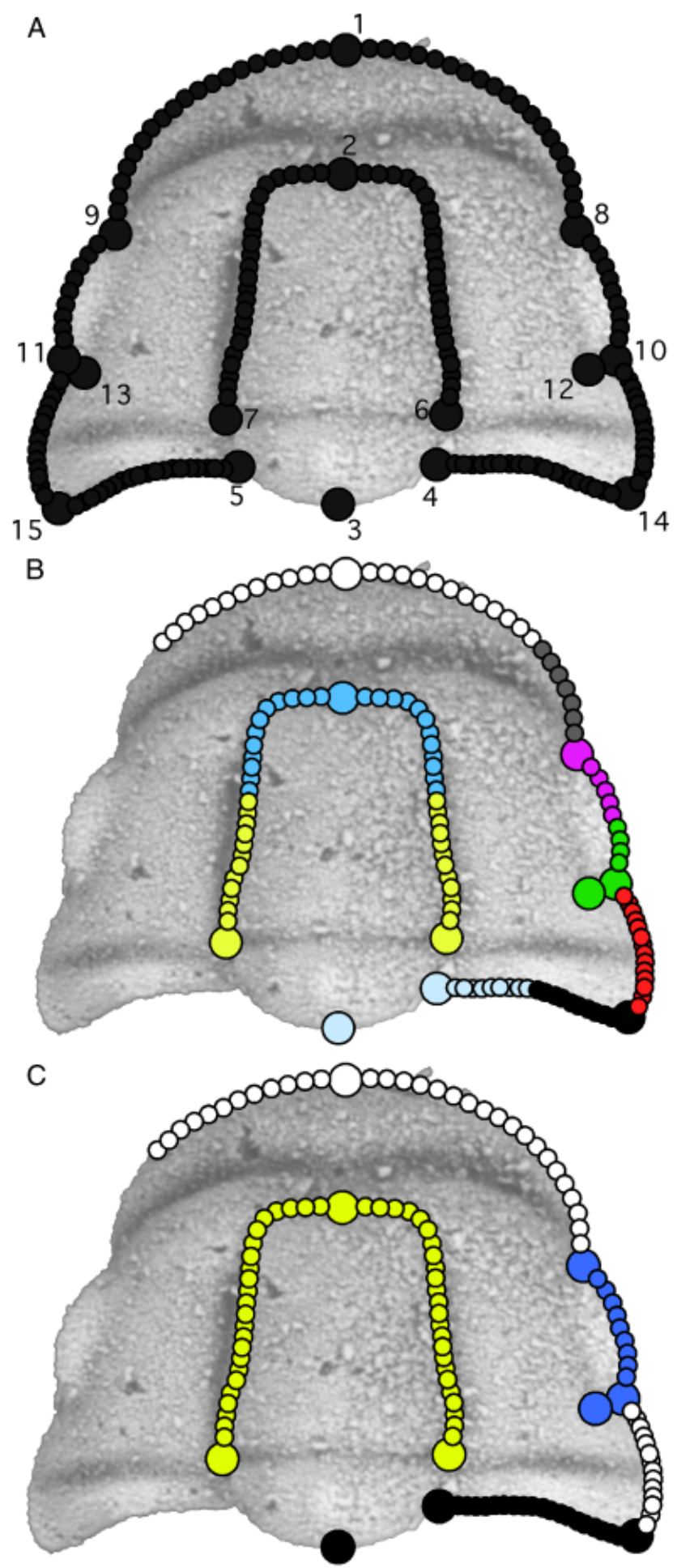

(Shergold and Cooper 2004). The carbonate was dissolved in weak acetic acid, and silicified sclerites were picked from the insoluble residue. Silicified cranidia were cleaned, blackened with dilute Indian ink, whitened with ammonium chloride, and mounted for photography using the standard orientation of Shaw (1957), with the dorsal surface of the palpebral lobes being positioned horizontally below a vertically mounted digital camera. Each cranidium was whitened, mounted, and photographed twice; approximately 2 weeks separated repeated imaging of each specimen.

The 72 cranidia included in the analyses presented herein range from 1.71 to $5.43 \mathrm{~mm}$ in sagittal length. Smaller cranidia were examined to confirm that only one species was represented in the sample (see also Webster in press), but were not included in the morphometric analysis because (1) they are more difficult to mount and photograph in a consistent orientation; (2) the potential for sampling a portion of ontogeny with a nonlinear pattern of allometry is greater when early ontogenetic stages are included; and (3) the potential for sampling a portion of ontogeny with an integration structure that differs from the mature condition is increased when earlier ontogenetic stages are included. Specimens are housed in the Field Museum of Natural History, Chicago (FMNH) and the Institute for Cambrian Studies, University of Chicago (ICS).

\section{Quantifying cranidial shape}

Landmark-based geometric morphometric methods (Rohlf and Slice 1990; Bookstein 1991; Dryden and Mardia 1998, Zelditch

Fig. 2. (A) Landmark and semilandmark selection on the cranidium of Crassifimbra? metalaspis. Landmarks (large circles, numbered): 1, Anterior cranidial margin on sagittal axis; 2, anterior of glabella on sagittal axis; 3, posterior margin of occipital ring on sagittal axis; 4, 5, intersection of occipital ring and posterior cranidial margin in dorsal view; 6,7 , intersection of SO with axial furrow; 8,9 , anterior tip of palpebral lobe; 10,11 , posterior tip of palpebral lobe; 12, 13, intersection of posterior branch of facial suture with distal margin of palpebral lobe in dorsal view; 14, 15; distal tip of posterior wing of fixigena. Semilandmarks (small circles, not numbered) summarize curvature of anterior cranidial margin and anterior branch of the facial suture (19 points each side between landmarks 1 and 8/9), distal margin of palpebral lobe (9 points each side between landmarks $8 / 9$ and $10 / 11$ ), posterior branch of the facial suture (14 points each side between landmarks $10 / 11$ and 14/15), posterior cranidial margin (19 points each side between landmarks $4 / 5$ and $14 / 15)$, and glabella anterior to SO (24 points each side between landmarks 2 and 6/7). See text for details. (B) The nine partitions of cranidial morphology analyzed herein: anterior glabella (blue); posterior glabella (yellow); anterior portion of the palpebral lobe (purple); posterior portion of the palpebral lobe (green); proximal posterior margin (pale blue); distal posterior margin (black); anterior margin (white); anterior branch of the facial suture (gray); and posterior branch of the facial suture (red). (C) Predicted modules in the cranidium based on the a priori functional hypothesis. Modules are associated with digestion/appendage attachment (glabella; yellow), the visual field (palpebral lobes; blue), ecdysis (facial sutures and anterior margin; white), and articulation between the cephalon and trunk (posterior margin; black). See text for details. 
et al. 2004; Webster and Sheets 2010) are widely used to study biological shape, shape variation, and covariation of shape with other biotic and abiotic variables. Such techniques have been applied in many paleontological studies (for trilobite examples, see Webster 2007 and references therein; Hopkins and Webster 2009; Webster in press). With sufficiently well-preserved material, the methods are capable of detecting even very subtle developmental signals in ancient organisms (Webster et al. 2001; Webster and Zelditch 2005, 2008; Webster 2007, in press). Such techniques are used herein to analyze the structure of integration within the cranidium of Crassifimbra? metalaspis.

The $x$ - and $y$-coordinates of 15 landmarks and 170 semilandmarks (along 10 curves) were digitized from images of 72 cranidia (Fig. 2A). Coordinate data were restricted to two dimensions because the cranidia are small in absolute size (making error associated with digitizing in the $z$-plane relatively large) and are of relatively low relief. It is assumed that exclusion of the third dimension does not markedly affect the conclusions. Landmark and outline coordinates were extracted using tpsDig2 (Rohlf 2009). Coordinates of sliding semilandmarks were calculated from the curve data using the SemiLand6 software (Sheets 2009), using the minimized Procrustes distance method to optimize their location along the outline. Measurement error associated with specimen mounting, photography, and digitizing replicability was negligible.

Allometry is a weak but significant contributor to cranidial shape variation in the sample (Fig. 3; multivariate regression of shape variables against the natural logarithm of centroid size [lnCS]: $\mathrm{SS}_{\text {total }}=0.0574 ; \mathrm{SS}_{\text {residual }}=0.0543 ; P<0.006$; significance determined by 1600 bootstraps). In order to remove the portion of shape variation resulting from allometry, the sample was computationally size standardized using Standard6 (Sheets 2001). This procedure involves conducting a linear regression of shape variables against $\operatorname{lnCS}$, then using this model to predict the shape of each specimen at a user-specified size. Residuals (shape deviations from the regression) remain associated with each specimen, so that the "size-standardized" shape of each specimen is the predicted shape of that specimen at the user-specified size plus its original residuals. When all specimens within a sample are size-standardized to the same $\operatorname{lnCS}$, shape variation is determined entirely by the residuals from the regression model. This technique is routinely used to remove the effects of allometry (Zelditch et al. 2004; Hopkins and Webster 2009; Webster in press). The full data set was standardized to a size of $\operatorname{lnCS}=3.7$, equivalent to a sagittal cranidial length of approximately $4.2 \mathrm{~mm}$. This is close to the maximum size (but within the size range) of sampled specimens, and therefore does not involve extrapolation of modeled ontogenetic vectors of shape change beyond the observed portion of ontogeny. Comparison of analyses with and without size-standardization revealed that this procedure had only a negligible effect on results (analyses not presented). This is not surprising, given the weak allometric signal in the data. All analyses presented below are based on the size-standardized data.

\section{Isolating integration resulting from direct interactions among developmental pathways}

The spatial structure of fluctuating asymmetry (FA; random deviations from bilateral symmetry) reveals which anatomical regions

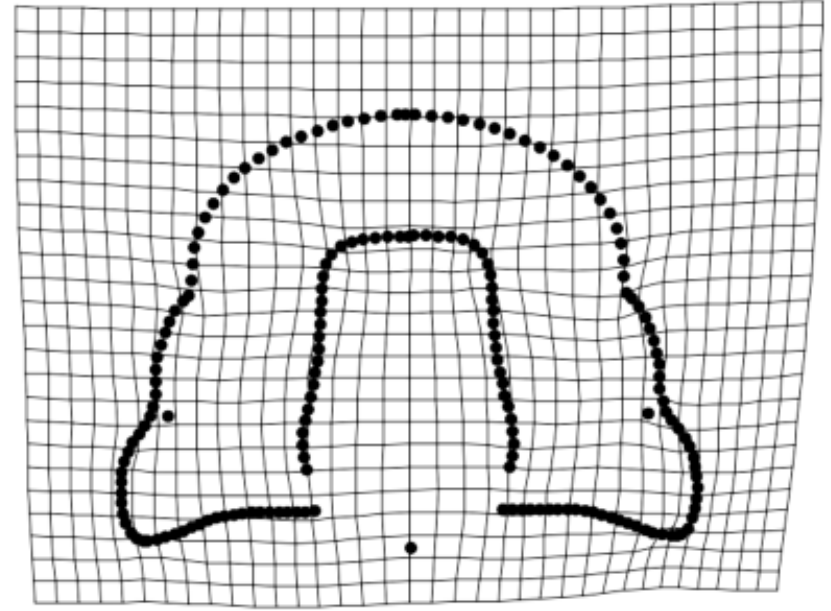

Fig. 3. Thin-plate spline deformation grid depicting shape change during the sampled portion of ontogeny of Crassifimbra? metalaspis. Landmark configuration shown in Fig. 2A. Reference form is consensus of all 72 configurations. The glabella proportionally narrows (tr.) anteriorly and widens (tr.) posteriorly, becoming more anteriorly tapered. The glabella also shortens slightly relative to cranidial length as the frontal area proportionally elongates (long.). The portion of the posterior cranidial margin distal to the fulcrum becomes less strongly posterolaterally oriented. The anterior cranidial margin and the anterior branch of the facial suture migrate away from the anterior margin of the glabella as the anterior cranidial border widens (tr. and long.). The anterior end of the palpebral lobe more or less initially contacts the anterior border furrow, but becomes separated from it as a strip of exoskeleton anterior to the ocular ridge elongates (long.). The palpebral lobe proportionally shortens (exsag.), and its distal edge (bounded by the facial suture) becomes slightly more strongly crescent-shaped.

are integrated through direct interactions among their respective morphogenetic developmental pathways. This is because the two sides of the body do not differ in the causes of parallel variation, that is, genetic or environmental factors acting independently in two or more modules (e.g., Klingenberg and Zaklan 2000; Klingenberg et al. 2001, 2003; Klingenberg 2005). Because the causes of parallel variation are held constant, these cannot be the cause of correlated FAs. Instead, correlated FAs arise from the processes that can transmit variation from one trait to another, thus inducing covariation between them; the source of such variation being a random perturbation of development. Therefore, correlations of (signed) asymmetries indicate integration due to direct interactions (e.g., Klingenberg and Zaklan 2000; Klingenberg et al. 2003; Klingenberg 2005; Zelditch et al. 2008, 2009). A particularly attractive feature of using correlated FAs to delimit modules is that the findings are less dependent on what happens to vary within a sample. This latter point is important because phenotypic integration results from the net effect of variation in multiple developmental processes that can overwrite each other (Hallgrímsson et al. 2007, 2009).

The presently favored approach for isolating the FA component of variation uses the two-factor mixed-model analysis of variance (ANOVA), with the two main effects being "Individuals" and "Sides" (Leamy 1984; Palmer and Strobeck 1986). The former 
effect quantifies the symmetric component of variation among individuals after correction for asymmetry; the latter effect quantifies the directional asymmetry between left and right sides of the organism. FA is quantified by the interaction between these two components, and its statistical significance is assessed by the F-ratio between the interaction mean square and measurement error mean square (Leamy 1984; Palmer and Strobeck 1986). This same approach has been adapted to Procrustes-based methods of shape analysis (Auffray et al. 1996; Klingenberg and McIntyre 1998; Klingenberg et al. 2002). A permutation test is used to determine the significance of the FA term. The SAGE software (Marquez 2007a) was used to conduct this analysis of FA and to generate shape coordinates for the Individuals and FA shape components for use in subsequent analyses.

\section{The among-partition correlation matrix}

To measure the correlations between the shape of cranidial regions we divided it into nine partitions (Fig. 2B): (1) anterior glabella; (2) posterior glabella; (3) anterior portion of the palpebral lobe; (4) posterior portion of the palpebral lobe; (5) proximal portion of the posterior cranidial margin (including a landmark at the midline of the posterior margin of the occipital ring); (6) distal portion of the posterior cranidial margin; (7) anterior cranidial margin; (8) anterior branch of the facial suture; and (9) posterior branch of the facial suture. The boundary between partitions (1) and (2) corresponds to the transition from the region of the glabella that proportionally narrows during ontogeny versus the region that proportionally widens during ontogeny (Fig. 3; Webster in press). The boundary between partitions (3) and (4) corresponds to a break in curvature on the distal margin of the palpebral lobe seen on some specimens, but is otherwise arbitrary. The boundary between partitions (5) and (6) corresponds to the location of the fulcrum (distal to which the cranidial margin is oriented more strongly posterolaterally and ventrally). The boundary between partitions (7) and (8) approximately corresponds to the contact between the anterior cranidial margin and the anterior branch of the facial suture, although this transition is often not marked by a distinct break in curvature.

The landmark (and semilandmark) coordinates for each partition were extracted from the whole; the shape of partitions (1), (2), and (7), displaying object symmetry, was summarized by landmarks on both sides of the sagittal axis (Fig. 2B). The shape of the other partitions, displaying matching symmetry, was summarized by landmarks on only one side of the cranidium (Fig. 2B). Each partition was subsequently treated as a separate multidimensional trait, and landmark configurations for each partition were superimposed separately. The pairwise Procrustes distances between all individuals were then calculated for each partition and the matrix correlation between distance matrices of partitions was calculated, yielding a matrix of correlations between shapes of the partitions. High correlation between two partitions indicates that shape deviation among specimens in one partition is associated with shape deviation among specimens in the other: that is, the partitions are structured similarly in their shape variation. The among-partition correlation matrix was calculated for both the Individuals and FA components of shape, permitting inference of the structure of symmetric phenotypic integration and of integration arising through direct interactions among developmental pathways, respectively. This matrix was computed using CORIANDIS (Marquez 2007b). The resultant correlation matrix was then assessed for its fit to the a priori model and also subjected to a series of exploratory analyses by hierarchical cluster analysis, reticulate network analysis, and graphical modeling.

\section{Hierarchical cluster analysis}

We use both Ward's (Ward 1963) and UPGMA (Farris 1969) ultrametric clustering methods to explore the correlation structure between the nine cranidial partitions. Such methods are widely used in integration studies (Zelditch et al. 2008, 2009, and references therein). Ward's method maximizes the hierarchical structure of the dendrogram by minimizing the variance of within-cluster distances. UPGMA clusters observations based on the mean distance between all objects in the cluster. The extent to which the clusters reconstruct the observed correlation matrix is assessed using the cophenetic correlation (Sneath and Sokal 1973), which measures the correlation between the distances between partitions in the observed data (calculated by subtracting each correlation from 1.0) and the distances between partitions on the cluster dendrogram. Values $<0.85$ indicate that distances are distorted on the dendrogram and that fit is poor. The assumption that clustering is actually hierarchical can be tested by the agglomerative coefficient (AC; Kaufman and Rousseeuw 1990; Zelditch et al. 2008), which quantifies the degree of hierarchical structure of the data. The $\mathrm{AC}$ is a function of the distance between each observation and the first cluster with which it is merged, divided by the dissimilarity between the last-merged objects. Values of AC range from 0 to 1 ; high values indicate much greater similarity among the first-merged observations than among the last-merged observations (i.e., strong hierarchy). Cophenetic correlations and ACs were calculated using the "cluster" package in R (Maechler et al. 2005; R Development Core Team 2008). Hierarchical clustering was performed in SPSS.

\section{Reticulate network analysis}

Standard hierarchical clustering methods are ultrametric, meaning that all distances between traits within a cluster are smaller than distances to traits outside the cluster and that all traits outside a cluster are equally far from all traits within. Reticulate network analysis is a nonultrametric approach that permits reticulations among clusters, such that a trait may belong to more than one cluster (Makarenkov and Legendre 2004; Makarenkov et al. 2004; Zelditch et al. 2008, 2009). Clustering is additive, relaxing the constraints on trait distances within and among clusters (Sattath and Tversky 1977). Reticulations are added if they significantly improve the additive dendrogram, based on an optimization between fit to the original distances and the number of linkages on the dendogram. Reticulate network analysis was conducted on the amongpartition correlation matrices using the T-REX software (Makarenkov 2000), using the conservative $Q_{1}$ optimization criterion for addition of reticulations (summarized by Zelditch et al. 2008, 2009).

\section{Graphical modeling}

Graphical modeling represents another method for exploring the associations between cranidial partitions (Magwene 2001; Young 
2004; Young and Hallgrímsson 2005; Polanski and Franciscus 2006; Allen 2008; Lawler 2008; Zelditch et al. 2009; but see criticisms by Mitteroecker and Bookstein 2007, 2009 and reply by Magwene 2009). An among-partition partial correlation matrix is computed by inverting the observed among-partition correlation matrix, then rescaling the values so that all elements along the diagonal are 1.0. When used in an exploratory fashion, a heuristic search is conducted to find a model containing the fewest edges that fits the data. Graphical modeling analyses were conducted using the MIM 3.2.0.7 (Edwards 2008). Heuristic searches were done in a stepwise fashion, using the options for a headlong search (i.e., edges inspected in random order), a bidirectional search (i.e., both adding and deleting edges), and an unrestricted search (i.e., allowing edges that were removed in a prior iteration to be reconsidered); for more details see the summary in Zelditch et al. (2009). An F-test was used to determine the significance of the resulting model given our relatively small sample size.

\section{Assessing the contribution of direct interactions to phenotypic integration}

The contribution of direct interactions to phenotypic integration can be estimated by comparing the covariance/correlation structure of the FA and Individuals components of variance (e.g., Klingenberg and Zaklan 2000; Klingenberg et al. 2003; Klingenberg 2005; Zelditch et al. 2008, 2009). Two such comparisons are made here. The first computes the covariance between landmarks for configurations representing the Individuals component of shape, and for configurations representing the FA component of shape. The structure of the two covariance matrices is then compared using a matrix correlation (the observed correlation being adjusted by multiplying it by the square root of the product of the repeatabilities of each covariance matrix). The significance of the adjusted matrix correlation is assessed using a Mantel test (1000 permutations). This analysis was performed in MACE (Marquez 2007c). The second comparison uses matrix correlation and a Mantel test to determine the similarity between the among-partition correlation matrices for the two components of variation. For both comparisons, significant correlation means that the structures of the Individuals and FA components of variation are more similar than expected by chance, indicating that direct interactions make a significant contribution to phenotypic integration.

\section{RESULTS}

\section{Isolating integration resulting from direct interactions among developmental pathways}

Results of the modified two-way ANOVA of the size-standardized data (Table 1) show that the Individuals component (relating to symmetric variation among specimens) accounts for $75 \%$ of the total shape variance. As expected for the bilaterally symmetrical cranidium, the Sides component (relating to directional asymmetry) accounts for a negligible proportion of total shape variance (smaller than measurement error). The Individuals $\times$ Sides interaction term, relating to FA, explains $20 \%$ of total shape variation and is highly significant. Measurement error (generated through inclusion of configurations digitized from replicate images of each specimen, above) is an order of magnitude smaller than FA.

\section{The among-partition correlation matrix}

The among-partition correlation matrix for symmetric variation among individuals is shown in Table 2 (lower triangle; derived from shape data of the Individuals component of variation, above). The anterior glabella partition significantly correlates (at 95\% confidence) with (1) posterior glabella, (2) both partitions along the posterior margin, the (3) anterior margin, and the (4) posterior branch of the facial suture partitions. The posterior glabella partition also significantly correlates with the proximal posterior margin and the anterior margin. Perhaps surprisingly, the anterior and posterior portions of the palpebral lobe do not significantly correlate with each other; indeed, the former shows significant correlation only with the partitions relating to the cranidial margin anterior to it, and the latter is not significantly correlated with any other partition. The proximal and distal portions of the posterior margin do not significantly correlate with each other; although both significantly correlate with the (1) anterior glabella, (2) posterior branch of the facial suture, and (3) anterior margin. The anterior margin significantly correlates with all partitions except the posterior portion of the palpebral lobe. The anterior and posterior branches of the

Table 1. Results of the modified two-way ANOVA of size-standardized shape data

\begin{tabular}{lcccrrr}
\hline Effect & SS & d.f. & MS & $F$-value & $P$-value & \% Variance explained \\
\hline Individuals & 0.16680 & 12,993 & 0.0000128 & 3.7020 & $<0.001$ & 75.497 \\
Sides & 0.00272 & 183 & 0.0000149 & 4.2879 & $<0.001$ & 1.232 \\
Individuals $\times$ Sides (FA) & 0.04506 & 12,993 & 0.0000035 & 13.3651 & $<0.001$ & 20.393 \\
Measurement error & 0.00636 & 26,352 & 0.0000002 & - & - & 2.879 \\
Total & 0.22094 & & & & 100.00000 \\
\hline
\end{tabular}

See text for details and interpretation.

$\mathrm{SS}$, sum of squares; d.f., degrees of freedom; MS, mean square. 
Table 2. Among-partition correlation matrix for symmetric phenotypic variation (derived from shape data of individuals component of variation; lower triangle) and for direct interactions among developmental pathways (derived from shape data of FA component of variation; upper triangle)

\begin{tabular}{lccccccccc}
\hline & Ant Glab & Post Glab & Ant Palp & Post Palp & Prox PM & Dist PM & Ant Marg & Ant Fac & Post Fac \\
\hline Ant Glab & & $\mathbf{0 . 4 3 2}$ & $\mathbf{0 . 1 9 2}$ & $\mathbf{0 . 2 0 7}$ & $\mathbf{0 . 1 8 6}$ & $\mathbf{0 . 5 3 9}$ & $\mathbf{0 . 1 8 9}$ & 0.149 & $\mathbf{0 . 3 5 2}$ \\
Post Glab & $\mathbf{0 . 2 3 9}$ & & 0.128 & $\mathbf{0 . 2 0 1}$ & $\mathbf{0 . 3 4 5}$ & $\mathbf{0 . 4 2 6}$ & $\mathbf{0 . 4 2 1}$ & $\mathbf{0 . 2 5 6}$ & $\mathbf{0 . 3 7 2}$ \\
Ant Palp & 0.117 & 0.100 & & $\mathbf{0 . 1 9 9}$ & 0.159 & 0.054 & 0.099 & 0.109 & 0.101 \\
Post Palp & 0.093 & 0.041 & 0.075 & & $\mathbf{0 . 4 4 9}$ & 0.144 & $\mathbf{0 . 4 3 0}$ & 0.116 & $\mathbf{0 . 2 0 6}$ \\
Prox PM & $\mathbf{0 . 2 2 9}$ & $\mathbf{0 . 3 4 3}$ & -0.042 & 0.019 & & $\mathbf{0 . 1 8 7}$ & $\mathbf{0 . 6 2 5}$ & 0.167 & $\mathbf{0 . 2 0 5}$ \\
Dist PM & $\mathbf{0 . 5 5 6}$ & 0.128 & 0.055 & 0.138 & 0.160 & & $\mathbf{0 . 2 8 6}$ & 0.183 & $\mathbf{0 . 5 6 4}$ \\
Ant Marg & $\mathbf{0 . 4 4 1}$ & $\mathbf{0 . 1 8 7}$ & $\mathbf{0 . 1 6 9}$ & 0.140 & $\mathbf{0 . 2 0 5}$ & $\mathbf{0 . 4 1 2}$ & & $\mathbf{0 . 1 9 2}$ & $\mathbf{0 . 3 5 3}$ \\
Ant Fac & 0.101 & 0.130 & $\mathbf{0 . 2 8 8}$ & 0.153 & 0.062 & 0.017 & $\mathbf{0 . 1 8 6}$ & & $\mathbf{0 . 3 2 5}$ \\
Post Fac & $\mathbf{0 . 6 2 9}$ & 0.175 & 0.077 & 0.144 & $\mathbf{0 . 2 5 2}$ & $\mathbf{0 . 5 6 4}$ & $\mathbf{0 . 5 4 6}$ & 0.100 & \\
\hline
\end{tabular}

Correlations significant at $95 \%$ confidence (based on 1000 permutations) are boldface and in shaded cells.

Locations of partitions shown in Fig. 2B. See text for details and interpretation.

Ant Glab, anterior glabella; Post Glab, posterior glabella; Ant Palp, anterior portion of palpebral lobe; Post Palp, posterior portion of palpebral lobe; Prox PM, proximal portion of posterior margin; Dist PM, distal portion of posterior margin; Ant Marg, anterior margin; Ant Fac, anterior branch of facial suture; Post Fac, posterior branch of facial suture.

facial suture are not significantly correlated with each other. The strongest correlations are found between the distal posterior margin and the posterior branch of the facial suture, between each of these partitions and the anterior glabella partition, and between the posterior branch of the facial suture and the anterior margin.

The among-partition correlation matrix for the FA component of variation (Table 2, upper triangle) is generally similar to that for the Individuals component. Most differences relate to the palpebral lobe partitions, which show significant correlation with each other and with the anterior glabella; the posterior portion of the palpebral lobe also significantly correlates with the (1) posterior glabella, (2) proximal posterior margin, (3) anterior margin, and the (4) posterior branch of the facial suture. The correlations between the anterior portion of the palpebral lobe and the anterior margin, and between the anterior portion of the palpebral lobe and the anterior branch of the facial suture, are not significant. The proximal and distal portions of the posterior margin show a weak but significant correlation. The posterior branch of the facial suture also significantly correlates with the posterior glabella and with the anterior branch of the facial suture. The strongest correlations are found between the distal posterior margin and the posterior branch of the facial suture, between the distal posterior margin and the anterior glabella, and between the proximal posterior margin and the anterior margin.

\section{Fitting a functional model to observed correlations}

Fit of the a priori functional hypothesis to the observed among-partition correlation structure is poor for both the Individuals and FA components of variation. The data deviate substantially from expectations of the model for both the Individuals $(\mathrm{AIC}=1854.388$, deviance $=98.107$, d.f. $=30$, $P<0.001)$ and $\mathrm{FA}$ components of variation $(\mathrm{AIC}=1850.433$, deviance $=128.947$, d.f. $=30, P<0.001)$. For both data sets, the $\triangle \mathrm{AIC}$ of the models relative to the fully saturated model is greater than zero, indicating exceptionally poor fit $(\triangle \mathrm{AIC}=38.107 ; \Delta \mathrm{AIC}=68.569$ for Individuals and $\mathrm{FA}$ components, respectively). The matrix correlations between the model and both data sets also reveal exceptionally poor fit of the model - the target and observed matrices are no more similar than expected by chance $\left(R_{\mathrm{M}}=0.046, P=0.32\right.$; $R_{\mathrm{M}}=0.050, P=0.36$ for the Individuals and FA components, respectively).

\section{Exploratory Analyses Hierarchical cluster analysis}

$\mathrm{AC}$ is $<0.53$ for dendrograms produced by both UPGMA and Ward's methods (Fig. 4), indicating that hierarchical structure is relatively weak. Dendrograms produced using the UPGMA method have a cophenetic correlation of $>0.85$, indicating satisfactory representation of the structure of the original among-partition correlation matrix. Ward's and UPGMA methods produce very similar clustering of partitions for the Individuals component of variation data (Fig. 4, A and B). Both identify a tight cluster consisting of the [[[anterior glabella+posterior branch of the facial suture] distal posterior margin] anterior margin] partitions. Both also identify clusters consisting of the [posterior glabella+proximal posterior margin] partitions, and the [[anterior palpebral lobe +anterior branch of the facial suture] posterior palpebral lobe] partitions. The second cluster is weakly linked to either the first or the third cluster (UPGMA or Ward's method, respectively). The two methods also produce reasonably similar clustering of partitions for the FA component of variation 
A

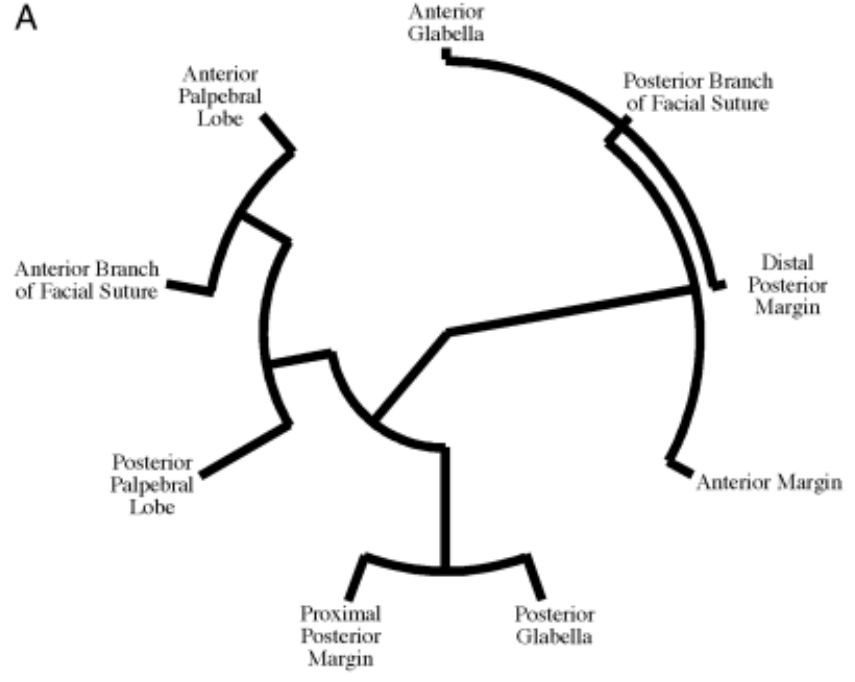

Individuals component, Ward's method

$(A C=0.522 ; C C=0.801)$

C

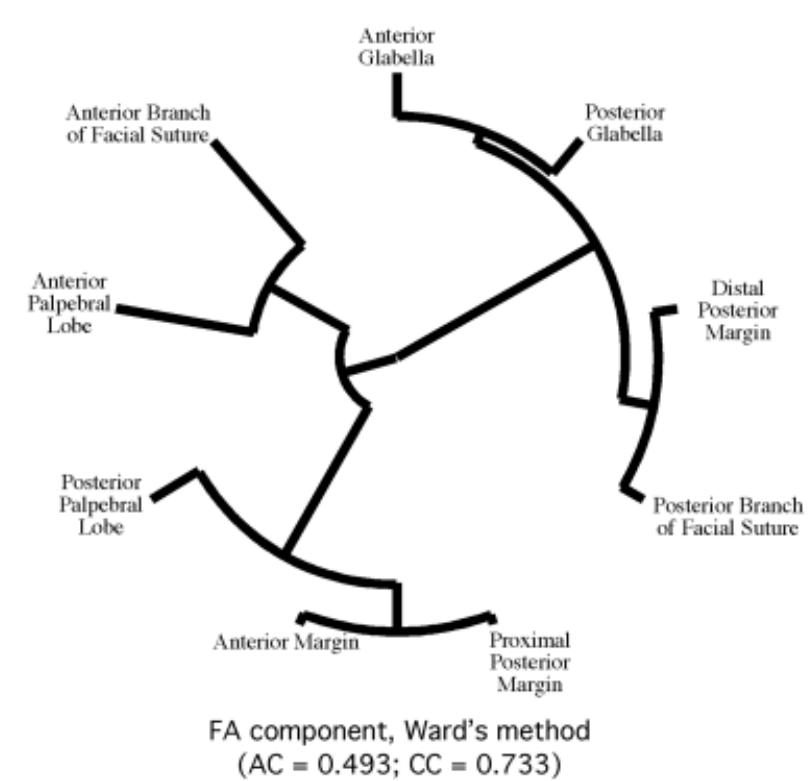

B

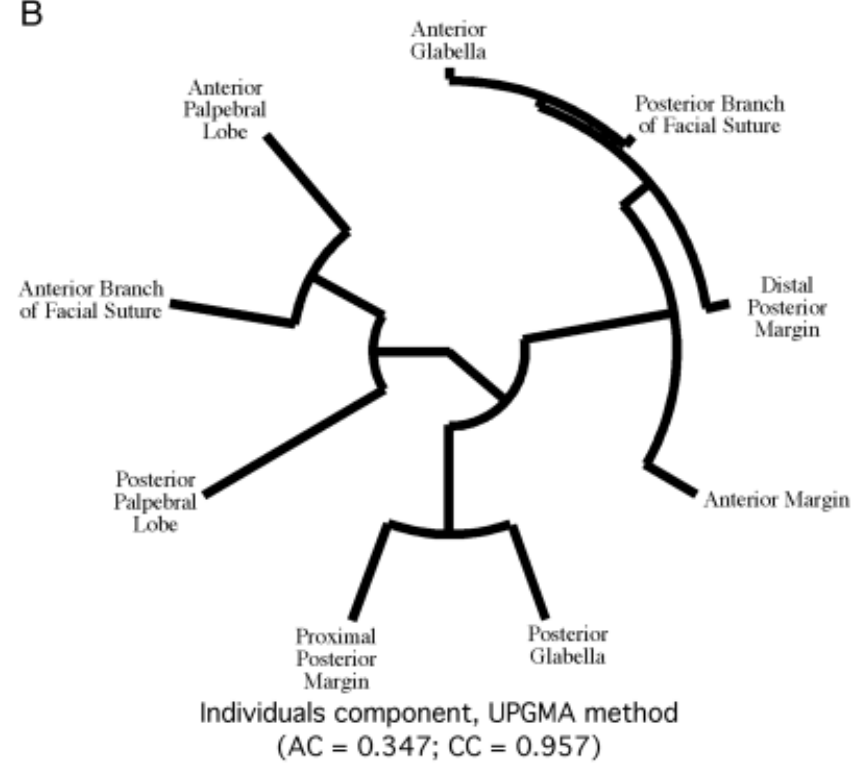

D

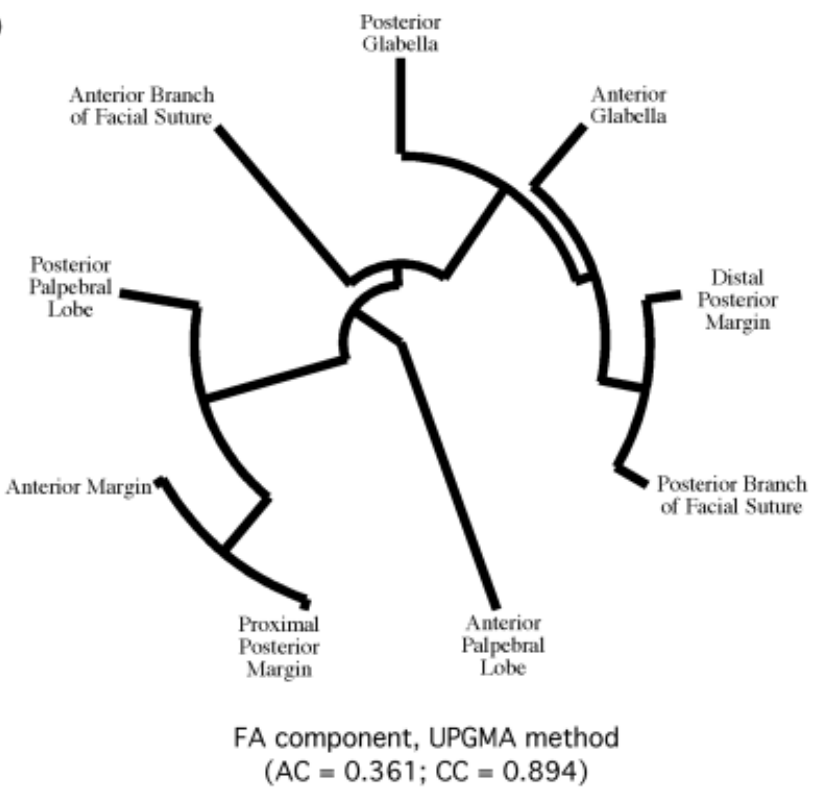

Fig. 4. Dendrograms depicting results of hierarchical cluster analyses of the among-partition correlation matrices for the Individuals (A, B) and fluctuating asymmetry (FA) (C, D) components of variation. (A, C), Ward's method. (B, D), UPGMA method. Root is central in each plot. AC, agglomerative coefficient; CC, cophenetic correlation. Location of partitions shown in Fig. 2B. See text for interpretation.

data (Fig. 4, C and D), although this clustering is not very similar to that for the Individuals component. For the FA data, both methods identify a tight cluster consisting of the [[distal posterior margin + posterior branch of the facial suture] anterior glabella + posterior glabella] partitions, although the methods differ in clustering relationships of the last two partitions to the first cluster. Both methods also identify a [[proximal posterior margin+anterior margin] posterior palpebral lobe] cluster. The anterior palpebral lobe and anterior branch of the facial suture partitions cluster very weakly with the second listed cluster (Ward's method), or the anterior branch of the facial suture partition forms an extremely weak cluster with the first listed cluster and the anterior palpebral lobe partition is not clustered with any other partitions (UPGMA).

\section{Reticulate network analysis}

The reticulogram for the Individuals component of variation identifies the same three clusters of partitions as did the hierarchical cluster analysis (Fig. 5A). However, reticulations 

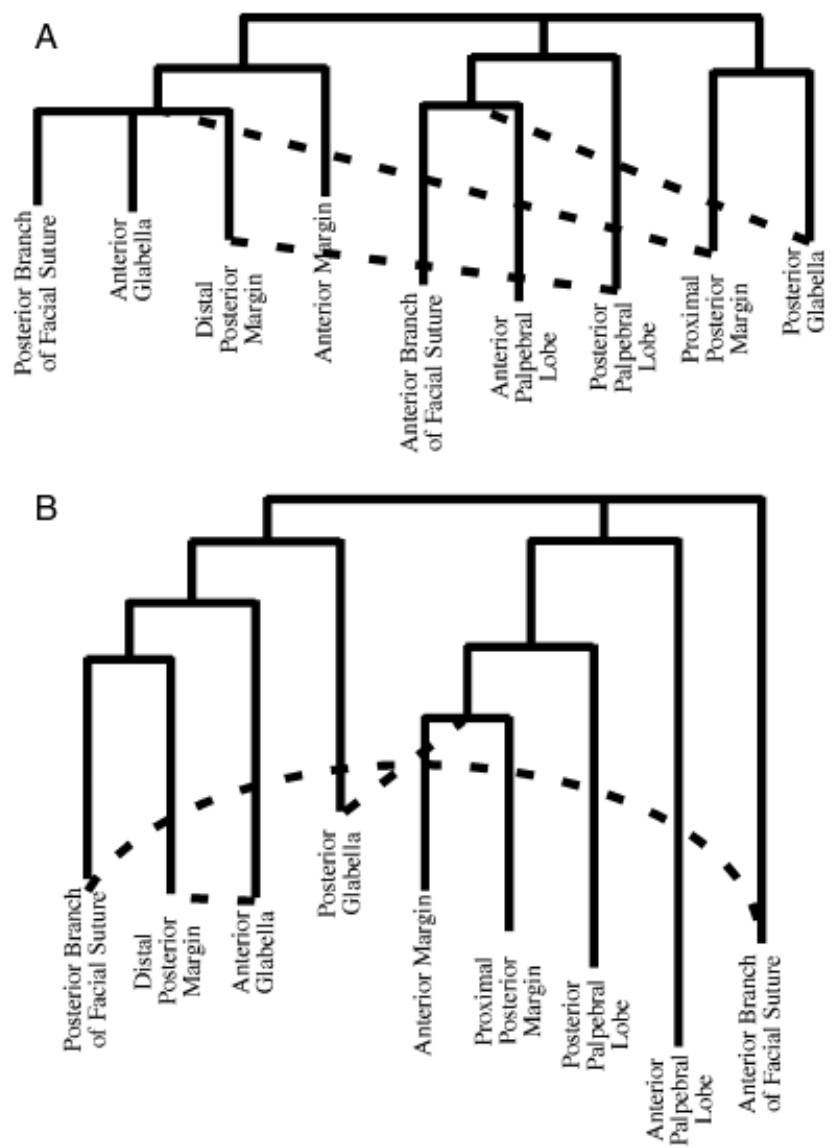

Fig. 5. Reticulograms depicting results of reticulate network analyses of the among-partition correlation matrices for the Individuals (A) and fluctuating asymmetry (B) components of variation. Location of partitions shown in Fig. 2B. See text for interpretation.

are added between the distal posterior margin and the posterior palpebral lobe partitions, between the proximal posterior margin partition and the [anterior glabella+distal posterior margin + posterior branch of the facial suture] cluster, and between the posterior glabella partition and the [anterior palpebral lobe+anterior branch of the facial suture] cluster (Fig. 5A). For the FA component, the reticulogram identifies the same two clusters of partitions as did the hierarchical cluster analysis (Fig. 5B). The anterior palpebral lobe partition clusters very weakly with the [[proximal posterior margin +anterior margin] posterior palpebral lobe] cluster. Reticulations are added between the anterior glabella and distal posterior margin partitions, and between the posterior glabella partition and the [proximal posterior margin +anterior margin] cluster (Fig. 5B). The anterior branch of the facial suture partition is linked to the posterior branch of the facial suture, but is otherwise isolated. It is surprising that so many reticulations are supported given that the cophenetic correlation is strong for both data sets.

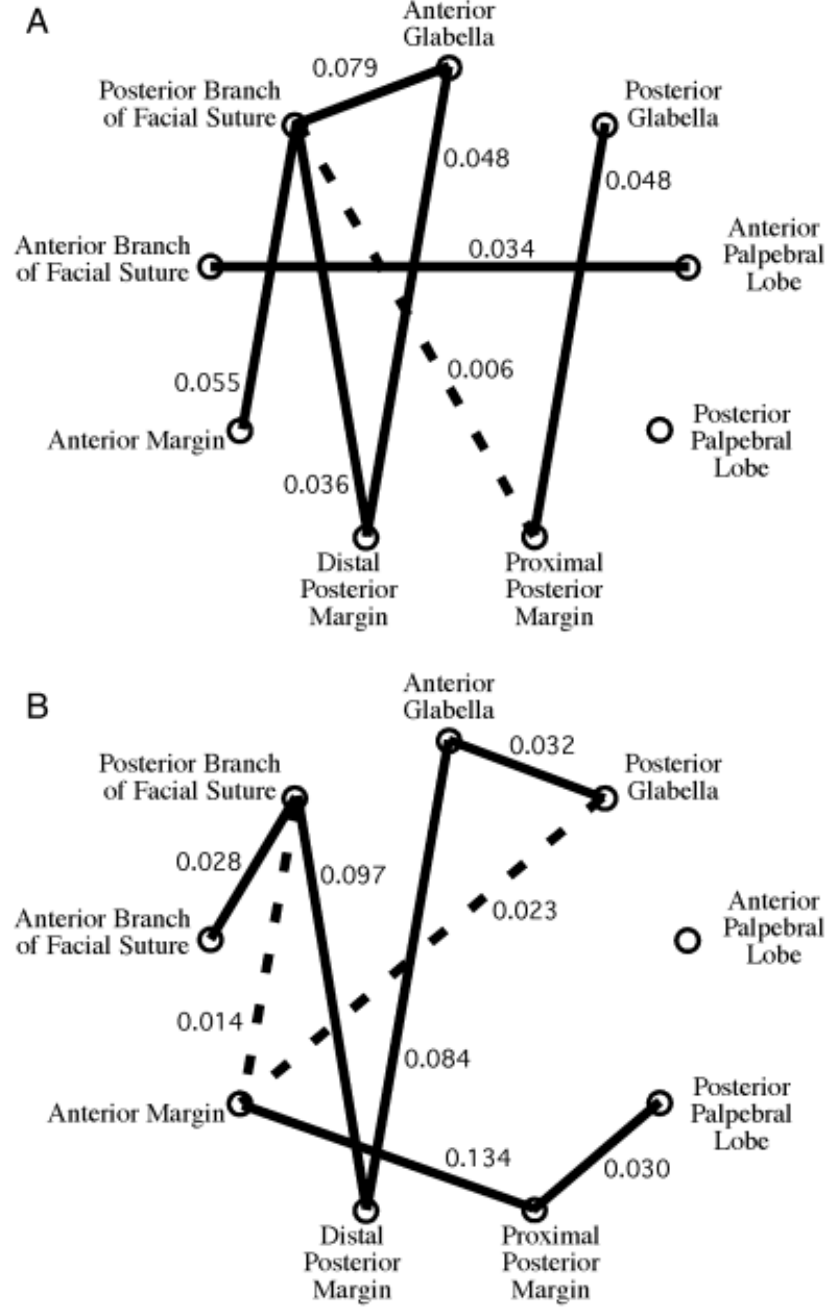

Fig. 6. Graphical models depicting edges recovered by exploratory analysis of among-partition partial correlation matrices for the Individuals (A) and fluctuating asymmetry (B) components of variation. Dotted lines indicate edges of relatively weak strength (adjacent to links). Location of partitions shown in Fig. 2B. See text for interpretation.

\section{Graphical modeling}

Graphical modeling of the Individuals component reveals an edge connecting the anterior palpebral lobe to the anterior branch of the facial suture, suggesting that these two form a single module (Fig. 6A). The posterior glabella and proximal posterior margin partitions are also connected, forming a second variational module. A third comprises the anterior glabella, distal posterior margin, and posterior branch of the facial suture partitions. The posterior branch of the facial suture is also connected to the anterior margin partition. The second and third putative modules are linked by an edge between the proximal posterior margin and the posterior branch of the facial suture, although the edge is weak. The posterior palpebral lobe is not linked to any other partition. 
Graphical modeling of the FA component reveals edges connecting the proximal posterior margin to both the posterior palpebral lobe and the anterior margin partitions (Fig. 6B). Edges are also discerned between the anterior glabella and posterior glabella partitions, between the anterior glabella and the distal posterior margin, between the distal posterior margin and the posterior branch of the facial suture, and between the anterior and posterior branches of the facial suture. These five partitions are therefore connected but each one is conditionally dependent on only one or two others. The first of the two modules is connected to the second by edges between the anterior margin and the posterior glabella partitions and between the anterior margin and the posterior branch of the facial suture partitions (both those edges, however, are relatively weak). The anterior palpebral lobe partition is not linked to any other partition.

\section{Inferred structure of phenotypic integration}

The a posteriori hypothesis of the structure of phenotypic integration inferred by congruence among the exploratory methods in their analyses of the Individuals component of variation is summarized in Fig. 7A. All methods support a module comprising the [anterior glabella+distal posterior margin + posterior branch of the facial suture + anterior margin] partitions, with tight integration among the first three partitions and somewhat weaker integration to the fourth. This module thus comprises traits that are conditionally dependent. Similarly, a module comprising the [posterior glabella+proximal posterior margin] partitions and a module comprising the [anterior palpebral lobe+anterior branch of the facial suture] partitions are consistently detected, indicating that these are also conditionally dependent. A weak association between the proximal posterior margin partition and the first listed module is detected by the methods that are not constrained to produce a strictly hierarchical structure, suggesting that these two parts are conditionally dependent. Hierarchical cluster analysis and reticulate network analysis find that the posterior palpebral lobe partition is very weakly integrated with the [anterior palpebral lobe+anterior branch of the facial suture] module, but the two are not conditionally dependent and are therefore are not detected by graphical modeling.

\section{Inferred structure of direct interactions among developmental pathways}

The a posteriori hypothesis for the modular structure of direct interactions is summarized in Fig. 7B. All exploratory methods suggest a module comprising the [proximal posterior margin+anterior margin+posterior palpebral lobe] partitions (with stronger integration between the first two partitions than to the third), and a module comprising the [distal posterior margin + posterior branch of the facial

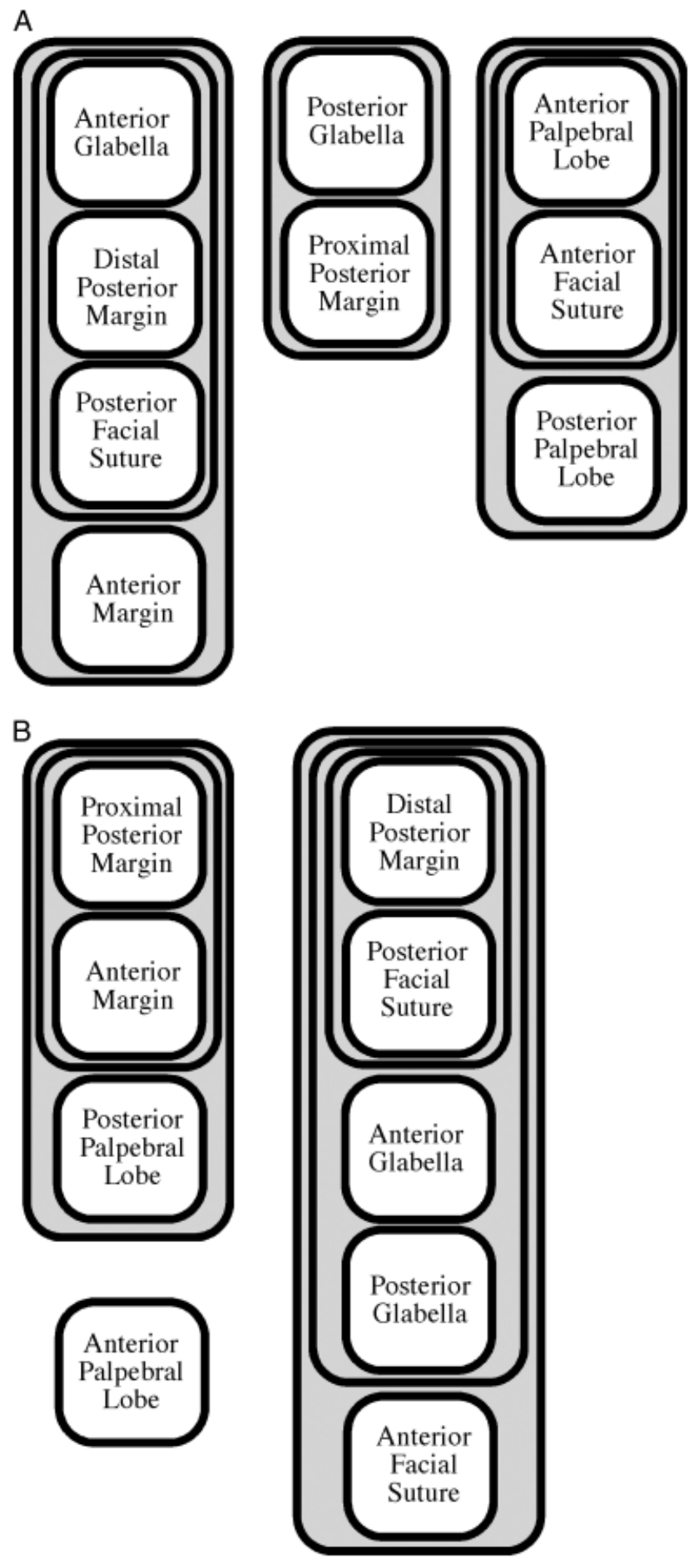

Fig. 7. Inferred structure of symmetric phenotypic integration (A) and of direct interactions among developmental pathways (B), based on congruence among exploratory analyses of the amongpartition correlation and partial correlation matrices (Figs. 4-6). Hierarchical integration structure among partitions is indicated by nested gray boxes. Location of partitions shown in Fig. 2B. See text for discussion. 
suture+anterior glabella+posterior glabella] partitions (with strongest integration between the first two listed partitions). Graphical modeling and reticulate network analysis find an association between the anterior branch of the facial suture and the posterior branch of the facial suture: this is consistent with the weak clustering of the anterior branch of the facial suture partition to the [distal posterior margin+posterior branch of the facial suture + anterior glabella + posterior glabella] module using the UPGMA method of hierarchical clustering. A weak association between the posterior glabella and anterior margin partitions is detected by the methods that are not constrained to produce a strictly hierarchical structure. The anterior palpebral lobe partition is grouped inconsistently by the different exploratory methods, and is here interpreted to be essentially isolated from all other partitions in terms of its FA (i.e., not integrated with any other partition).

\section{Assessing the contribution of direct interactions to phenotypic integration}

The landmark covariance structures of the Individuals and the FA components of shape are only weakly associated (adjusted matrix correlation $=0.225$ ), although they are more similar than expected by chance $(P=0.001$ based on Mantel test, 1000 bootstraps). Matrix correlation between the amongpartition correlation matrices for the Individuals and FA components of shape is significantly higher than expected by chance $\left(R_{\mathrm{M}}=0.448, P=0.02\right.$ based on Mantel test, 1000 permutations).

\section{DISCUSSION}

Functional association is a poor predictor of correlation (and therefore integration) among cranidial partitions in this trilobite. The functional hypothesis shows a poor fit to the observed among-partition associations for the Individuals component of variation and an even worse fit to those for the FA component of variation. Other than an association between the posterior branch of the facial suture and anterior margin partitions and between the anterior and posterior palpebral lobe partitions, none of the predicted strong correlations between partitions made by this hypothesis are observed for the Individuals component of shape variation (compare Figs. 2C and 7A). Only the predicted correlations between the anterior and posterior glabella partitions, and between the anterior and posterior branches of the facial suture partitions, are met by the observed data for the FA component of variation (compare Figs. 2C and 7B). The exploratory analyses reveal a pattern strikingly different from the one anticipated by function: integrated cranidial regions almost invariably are neighboring partitions, suggesting that integration arises from localized morphogenetic controls. Neighboring, integrated partitions also share similar patterns of ontogenetic shape change (Fig. 3), offering further support for the importance of localized morphogenetic controls to cranidial shape variation in this trilobite. Each common morphogenetic control likely involved a strong component of parallel variation because direct interactions contribute little to phenotypic integration; the correlation between the structure of the Individuals and FA components of variation is relatively weak even if the matrices are more similar than expected by chance. The inferred structures of phenotypic integration and of direct interactions are not particularly similar (Fig. 7, A and B). Although the distal posterior margin, posterior branch of the facial suture, and anterior glabella partitions are tightly integrated in both data sets, the associations between other partitions differ. Parallel variation among partitions is therefore interpreted to have strongly influenced the structure of phenotypic integration in the cranidium of this trilobite, modifying or masking most integration patterns resulting from direct interactions among developmental pathways.

The structure of cranidial integration that we find here suggests that there are localized morphogenetic controls on cranidial form, but localized morphogenetic processes do not explain the integration between the [anterior glabella + anterior margin] and [distal posterior margin + posterior branch of the facial suture] groups of partitions (Fig. 7A). This unanticipated correlation highlights the fact that we still have much to learn regarding processes regulating trilobite morphology. It is possible that the structure of integration detected herein was complicated by the cumulative effects of multiple processes, perhaps operating at different ontogenetic stages (the palimpsest model of Hallgrímsson et al. 2007). The amongpartition correlation structure for the Individuals component of variation is stable over the portion of ontogeny analyzed here (data not shown), so any such change in the structure of integration would have to have occurred at an earlier ontogenetic stage. Improved understanding of trilobite paleobiology might lead to generation of novel a priori hypotheses that explain some or all aspects of integration. For example, models of Hox gene expression domains have been proposed for the axial region of trilobites (Sundberg 2000; Hughes 2003a, b): a hypothesis for modularity based on Hox expression domains could be applied to the whole cranidium if we had more complete knowledge of the lateral course of segments away from the glabella.

That we find parallel variation to be the dominant source of phenotypic integration is important because integration arising through parallel variation is hypothesized to be more evolutionarily labile than integration arising through direct interactions among developmental pathways (Klingenberg 2005). That lability, and its consequences for phenotypic diversification, could explain why early ptychoparioid lineages 
are hard to consistently diagnose (summarized in Webster in press): frequent change in the structure of integration would relax long-term constraints on the directions of phenotypic evolution. Whether trilobite lineages do indeed show labile patterns of integration, and whether that lability increases or decreases over time, are open and important questions for understanding the dynamics of diversification. This is the first study to investigate modularity in a trilobite (indeed, in any ancient arthropod), and it is encouraging that a (largely sensible) structure of variational and developmental modularity can be resolved on these exquisitely preserved fossils despite approximately 510 million years of entombment. The fossil record can thus be used to investigate the stability of the structure of integration through deep time, to calibrate the duration of those structures, and most importantly, to investigate the impact of integration on the dynamics of diversity.

\section{Acknowledgments}

H. D. Sheets generously fixed a software bug at very short notice. Assistance in the field during collection of the material studied here was provided by R. R. Gaines, L. McCollum, and M. McCollum. The manuscript was improved by comments from two anonymous reviewers.

\section{REFERENCES}

Ackermann, R. R., and Cheverud, J. M. 2000. Phenotypic covariance structure in tamarins (genus Saguinus): a comparison of variation patterns using matrix correlation and common principal component analysis. Am. J. Phys. Anthropol. 111: 489-501.

Akaike, H. 1974. A new look at the statistical model identification. IEEE Trans. Automat. Contr. 19: 716-723.

Allen, C. E. 2008. The "eyespot" module and eyespots and modules: development, evolution, and integration of a complex phenotype. J. Exp. Zool., Part B: Mol. Dev. Evol. 310B: 179-190.

Auffray, J.-C., Alibert, P., Renaud, S., Orth, A., and Bonhomme, F. 1996. Fluctuating asymmetry in Mus musculus subspecific hybridization: traditional and procrustes comparative approaches. In L. F. Marcus, M. Corti, A. Loy, G. J. P. Naylor, and D. E. Slice (eds.). Nato ASI Series, Series A: Life Science. Plenum Press, New York and London. pp. 275-284.

Bookstein, F. L. 1991. Morphometric Tools for Landmark Data: Geometry and Biology. Cambridge University Press, Cambridge, UK. 435 pp.

Burger, R. 1986. Constraints for the evolution of functionally coupled characters: a nonlinear analysis of a phenotypic model. Evolution 40: 182-193.

Cheverud, J. M. 1982. Phenotypic, genetic, and environmental morphological integration in the cranium. Evolution 36: 499-516.

Cheverud, J. M. 1984. Quantitative genetics and developmental constraints on evolution by selection. J. Theor. Biol. 110: 155-171.

Cheverud, J. M. 1995. Morphological integration in the saddle-back tamarin (Saguinus fuscicollis) cranium. Am. Nat. 145: 63-89.

Cheverud, J. M., Hartman, S. E., Richtsmeier, J. T., and Atchley, W. R. 1991. A quantitative genetic analysis of localized morphology in mandibles of inbred mice using finite-element scaling analysis. J. Craniofac. Genet. Dev. Biol. 11: 122-137.

Cheverud, J. M., Wagner, G. P., and Dow, M. M. 1989. Methods for the comparative analysis of variation patterns. Syst. Zool. 38: 201-213.

Dietz, E. J. 1983. Permutation tests for association between two distance matrices. Syst. Zool. 32: 21-26.

Drake, A. G., and Klingenberg, C. P. 2010. Large-scale diversification of skull shape in domestic dogs: disparity and modularity. Am. Nat. 175: 289-301.
Dryden, I. L., and Mardia, K. V. 1998. Statistical Shape Analysis. John Wiley and Sons, Chichester, UK. 347 pp.

Edwards, D. 2008. MIM: A Program for Graphical Modeling. Version 3.2.0.7. Hypergraph Software. Available at www.hypergraph.dk/. Accessed May 19, 2009.

Farris, J. S. 1969. On the cophenetic correlation coefficient. Syst. Zool. 18: 279-285

Foote, M. 1989. Perimeter-based Fourier analysis: a new morphometric method applied to the trilobite cranidium. J. Paleontol. 63: 880-885.

Foote, M. 1990. Nearest-neighbor analysis of trilobite morphospace. Syst. Zool. 39: 371-382.

Foote, M. 1991. Morphologic patterns of diversification: examples from trilobites. Palaeontology 34: 461-485.

Foote, M. 1993a. Discordance and concordance between morphological and taxonomic diversity. Paleobiology 19: 185-204.

Foote, M. 1993b. Contributions of individual taxa to overall morphological disparity. Paleobiology 19: 403-419.

Fortey, R. A. 2001. Trilobite systematics: the last 75 years. J. Paleontol. 75: 1141-1151.

Hallgrímsson, B., et al. 2009. Deciphering the palimpsest: studying the relationship between morphological integration and phenotypic covariation. Evol. Biol. 36: 355-376.

Hallgrímsson, B., Lieberman, D. E., Liu, W., Ford-Hutchinson, A. F., and Jirik, F. R. 2007. Epigenetic interactions and the structure of phenotypic variation in the cranium. Evol. Dev. 9: 76-91.

Hallgrímsson, B., Willmore, K., Dorval, C., and Cooper, D. M. L. 2004. Craniofacial variability and modularity in macaques and mice. J. Exp. Zool., Part B: Mol. Dev. Evol. 302B: 207-225.

Hopkins, M. J., and Webster, M. 2009. Ontogeny and geographic variation of a new species of the corynexochine trilobite Zacanthopsis (Dyeran, Cambrian). J. Paleontol. 83: 524-547.

Hughes, N. C. 2003a. Trilobite body patterning and the evolution of arthropod tagmosis. BioEssays 25: 386-395.

Hughes, N. C. 2003b. Trilobite tagmosis and body patterning from morphological and developmental perspectives. Integr. Comp. Biol. 43 185-206.

Hunt, G. 2007. Evolutionary divergence in directions of high phenotypic variance in the ostracode genus Poseidonamicus. Evolution 61: 1560-1576.

Kaufman, L., and Rousseeuw, P. 1990. Finding Groups in Data, An Introduction to Cluster Analysis. Wiley, New York.

Klingenberg, C. P. 2004. Integration, modules, and development: molecules to morphology to evolution. In M. Pigliucci and K. A. Preston (eds.). Phenotypic Integration: Studying the Ecology and Evolution of Complex Phenotypes. Oxford University Press, Oxford, pp. 213-230.

Klingenberg, C. P. 2005. Developmental constraints, modules, and evolvability. In B. Hallgrimsson and B. K. Hall (eds.). Variation: A Central Concept in Biology. Elsevier Academic Press, San Diego, pp. 219-248.

Klingenberg, C. P., Badyaev, A. V., Sowry, S. M., and Beckwith, N. J. 2001. Inferring developmental modularity from morphological integration: analysis of individual variation and asymmetry in bumblebee wings. Am. Nat. 157: 11-23.

Klingenberg, C. P., Barluenga, M., and Meyer, A. 2002. Shape analysis of symmetric structures: quantifying variation among individuals and asymmetry. Evolution 56: 1909-1920.

Klingenberg, C. P., Leamy, L. J., and Cheverud, J. M. 2004. Integration and modularity of quantitative trait locus effects on geometric shape in the mouse mandible. Genetics 166: 1909-1921.

Klingenberg, C. P., and McIntyre, G. S. 1998. Geometric morphometrics of developmental instability: analyzing patterns of fluctuating asymmetry with procrustes methods. Evolution 52: 1363-1375.

Klingenberg, C. P., Mebus, K., and Auffray, J. C. 2003. Developmental integration in a complex morphological structure: how distinct are the modules in the mouse mandible? Evol. Dev. 5: 522-531.

Klingenberg, C. P., and Zaklan, S. D. 2000. Morphological integration between developmental compartments in the Drosophila wing. Evolution 54: 1273-1285.

Kohn, L. A. P., and Atchley, W. R. 1988. How similar are genetic correlation structures - data from mice and rats. Evolution 42: 467-481. 
Lawler, R. R. 2008. Morphological integration and natural selection in the postcranium of wild Verreaux's sifaka (Propithecus verreauxi verreauxi). Am. J. Phys. Anthropol. 136: 204-213.

Leamy, L. 1984. Morphometric studies in inbred and hybrid house mice. 5. Directional and fluctuating asymmetry. Am. Nat. 123: 579-593.

Maechler, M., Rousseeuw, P., Struyf, A., and Hubert, M. 2005. Cluster analysis basics and extensions. Available at http://cran.r-project.org/web/ packages/cluster/

Magwene, P. M. 2001. New tools for studying integration and modularity. Evolution 55: 1734-1745.

Magwene, P. M. 2009. Statistical methods for studying modularity: a reply to Mitteroecker and Bookstein. Syst. Biol. 58: 146-149.

Makarenkov, V. 2000. T-REX. Version 4.0a1. Available at http://www. labunix.uqam.ca/ makarenv/trex.html. Accessed May 19, 2009.

Makarenkov, V., and Legendre, P. 2004. From a phylogenetic tree to a reticulated network. J. Comput. Biol. 11: 195-212.

Makarenkov, V., Legendre, P., and Desdevises, Y. 2004. Modelling phylogenetic relationships using reticulated networks. Zool. Scr. 33: 89-96.

Mantel, N. 1967. The detection of disease clustering and a generalized regression approach. Cancer Res. 27: 209-220.

Marquez, E. 2007a. SAGE, version 1.03. Available at http://www-personal. umich.edu/ emarquez/morph/index.html. Accessed May 20, 2009.

Marquez, E. 2007b. CORIANDIS. Available at http://www-personal. umich.edu/ emarquez/morph/index.html. Accessed May 20, 2009.

Marquez, E. 2007c. MACE. Available at http://www-personal.umich.edu/ $\sim$ emarquez/morph/index.html. Accessed May 20, 2009.

Marroig, G., and Cheverud, J. M. 2005. Size as a line of least evolutionary resistance: diet and adaptive morphological radiation in new world monkeys. Evolution 59: 1128-1142.

Mitteroecker, P., and Bookstein, F. 2007. The conceptual and statistical relationship between modularity and morphological integration. Syst. Biol. 56: 818-836.

Mitteroecker, P., and Bookstein, F. L. 2009. Examining modularity via partial correlations: a rejoinder to a comment by Paul Magwene. Syst. Biol. 58: 346-348.

Monteiro, L. R., Bonato, V., and dos Reis, S. F. 2005. Evolutionary integration and morphological diversification in complex morphological structures: mandible shape divergence in spiny rats (Rodentia, Echimyidae). Evol. Dev. 7: 429-439.

Olson, E. C., and Miller, R. L. 1958. Morphological Integration. University of Chicago Press, Chicago, 355 pp [Reprinted with a new Afterword by B. Chernoff and P. M. Magwene, 1999.].

Palmer, A. R., and Strobeck, C. 1986. Fluctuating asymmetry-Measurement, analysis, patterns. Annu. Rev. Ecol. Syst. 17: 391-421.

Polanski, J. M., and Franciscus, R. G. 2006. Patterns of craniofacial integration in extant Homo, Pan, and Gorilla. Am. J. Phys. Anthropol. 131: 38-49.

R Development Core Team. 2008. R: A Language and Environment for Statistical Computing Version 2.6.2. R Foundation for Statistical Computing, Vienna, Austria. Available at http://www.r-project.org

Renaud, S., Auffray, J.-C., and Michaux, J. 2006. Conserved phenotypic variation patterns, evolution along lines of least resistance, and departure due to selection in fossil rodents. Evolution 60: 1701-1717.

Rohlf, F. J. 2009. tpsDig. Version 2.14. Department of Ecology and Evolution, State University of New York, Stony Brook. Available at http:// life.bio.sunysb.edu.morph/

Rohlf, F. J., and Slice, D. 1990. Extensions of the Procrustes method for the optimal superimposition of landmarks. Syst. Zool. 39: 40-59.

Sattath, S., and Tversky, A. 1977. Additive similarity trees. Psychometrika 42: $319-345$

Schlosser, G., and Wagner, G. P. (eds.). 2004. Modularity in Development and Evolution. University of Chicago Press, Chicago. $600 \mathrm{pp}$.

Schluter, D. 1996. Adaptive radiation along genetic lines of least resistance. Evolution 50: 1766-1774.

Shaw, A. B. 1957. Quantitative trilobite studies II. Measurement of the dorsal shell of non-agnostidean trilobites. J. Paleontol. 31: 193207.
Sheets, H. D. 2001. Standard6beta. Department of Physics, Canisius College, Buffalo, New York. Available at http://www.canisius.edu/ sheets/ morphsoft.html

Sheets, H. D. 2009. SemiLand6. 7th Beta Version. Department of Physics, Canisius College, Buffalo, New York. Available at http://www.canisius. edu/ sheets/morphsoft.html

Shergold, J. H., and Cooper, R. A. 2004. The Cambrian period. In F. M. Gradstein, J. G. Ogg, and A. G. Smith (eds.). A Geologic Time Scale 2004. Cambridge University Press, UK, pp. 147-164.

Simpson, G. G. 1944. Tempo and Mode in Evolution. Columbia University Press, New York. 237 pp.

Sneath, P. H. A., and Sokal, R. R. 1973. Numerical Taxonomy. W. H. Freeman, San Francisco.

Sniegowski, P. D., and Murphy, H. A. 2006. Evolvability. Curr. Biol. 16: R831-R834.

Sundberg, F. A. 2000. Homeotic evolution in Cambrian trilobites. Paleobiology 26: 258-270.

Sundberg, F. A., and McCollum, L. B. 2000. Ptychopariid trilobites of the Lower-Middle Cambrian boundary interval, Pioche Shale, southeastern Nevada. J Paleontol. 74: 604-630.

Wagner, G. P. 1988. The influence of variation and of developmental constraints on the rate of multivariate phenotypic evolution. J. Evol. Biol. 1 $45-66$.

Wagner, G. P., and Altenberg, L. 1996. Complex adaptations and the evolution of evolvability. Evolution 50: 967-976.

Ward, J. H. Jr 1963. Hierarchical grouping to optimize an objective function. J. Am. Stat. Assoc. 58: 236-244.

Webster, M. 2007. Ontogeny and evolution of the Early Cambrian trilobite genus Nephrolenellus (Olenelloidea). J. Paleontol. 81: 1168-1193.

Webster, M. In press. The structure of cranidial shape variation in three early ptychoparioid trilobite species from the Dyeran-Delamaran (traditional "Lower-Middle" Cambrian) boundary interval of Nevada, USA. J. Paleontol.

Webster, M., and Sheets, H. D. 2010. A practical introduction to landmarkbased geometric morphometrics. In J. Alroy and G. Hunt (eds.). Quantitative Methods in Paleobiology. Paleontological Society Papers. Vol. 16. Published by the Paleontological Society and printed by Yale University Printing and Publishing Services, New Haven, CT. pp. 163-188.

Webster, M., Sheets, H. D., and Hughes, N. C. 2001. Allometric patterning in trilobite ontogeny: testing for heterochrony in Nephrolenellus. In M. L. Zelditch (ed.). Beyond Heterochrony: The Evolution of Development. Wiley and Sons, New York, pp. 105-144.

Webster, M., and Zelditch, M. L. 2005. Evolutionary modifications of ontogeny: heterochrony and beyond. Paleobiology 31: 354-372.

Webster, M., and Zeldtich, M. L. 2008. Integration and regulation of developmental systems in trilobites. In I. Rábano, R. Gozalo, and D. García-Bellido (eds.). Advances in Trilobite Research. Cuadernos del Museo Geominero 9. Instituto Geológico y Minero de España, Madrid, pp. 427-433.

Whittington, H. B., et al. 1997. Treatise on Invertebrate Paleontology. Part O. Arthropoda 1. Trilobita, Revised. Volume 1: Introduction, Order Agnostida, Order Redlichiida. Geological Society of America and Univeristy of Kansas, Boulder, Colorado and Lawrence, Kansas. 530 pp.

Young, N. 2004. Modularity and integration in the hominoid scapula. J. Exp. Zool., Part B: Mol. Dev. Evol. 302B: 226-240.

Young, N. M., and Hallgrímsson, B. 2005. Serial homology and the evolution of mammalian limb covariation structure. Evolution 59: 2691-2704.

Zelditch, M. L., Swiderski, D. L., Sheets, H. D., and Fink, W. L. 2004. Geometric Morphometrics of Biologists: A Primer. Elsevier Academic Press, San Diego. 443 pp.

Zelditch, M. L., Wood, A. R., Bonett, R. M., and Swiderski, D. L. 2008. Modularity of the rodent mandible: integrating bones, muscles, and teeth. Evol. Dev. 10: 756-768.

Zelditch, M. L., Wood, A. R., and Swiderski, D. L. 2009. Building developmental integration into functional systems: function-induced integration of mandibular shape. Evol. Biol. 36: 71-87. 